\title{
Open
}

\section{Fragile $X$ full mutation expansions are inhibited by one or more AGG interruptions in premutation carriers}

\author{
Sarah L. Nolin, PhD ${ }^{1}$, Anne Glicksman, MS¹, Nicole Ersalesi, BS ${ }^{1}$, Carl Dobkin, PhD ${ }^{1}$, W. Ted Brown, MD, \\ $\mathrm{PhD}^{1}$, Ru Cao, PhD², Eliot Blatt², Sachin Sah, MS², Gary J. Latham, PhD² and Andrew G. Hadd, PhD²
}

Purpose: Fragile X CGG repeat alleles often contain one or more AGG interruptions that influence allele stability and risk of a full mutation transmission from parent to child. We have examined transmissions of maternal and paternal alleles with 45-90 repeats to quantify the effect of AGG interruptions on fragile $\mathrm{X}$ repeat instability.

Methods: A novel FMR1 polymerase chain reaction assay was used to determine CGG repeat length and AGG interruptions for 1,040 alleles from 705 families.

Results: We grouped transmissions into nine categories of five repeats by parental size and found that in every size category, alleles with no AGGs had the greatest risk for instability. For maternal alleles $<75$ repeats, $89 \%(24 / 27)$ that expanded to a full mutation had no

\section{INTRODUCTION}

Fragile X syndrome (FXS; OMIM 300624) is one of more than 40 repeat disorders characterized by repeat instability on transmission from parent to child. ${ }^{1}$ For the fragile $\mathrm{X}$ gene $F M R 1$, the repeat is a CGG trinucleotide located in the $5^{\prime}$ untranslated region that can expand to more than 200 copies (the full mutation) on maternal transmission. ${ }^{2-4}$ Full mutation alleles are silenced by a process analogous to $\mathrm{X}$ inactivation, which results in the absence of the gene product, FMRP, and the FXS in males and some females. ${ }^{5}$ Although the repeat is highly stable when transmitted from individuals with normal alleles (6-44 CGGs), it is remarkably unstable on maternal transmission of premutation alleles (55-200 CGGs), which frequently expand to the full mutation in one generation. This risk of full mutation expansion increases with maternal CGG repeat length to nearly $100 \%$ for mothers with $>90$ CGGs. ${ }^{6}$ With a reported frequency of 1 in $150-250$ premutation alleles in females in the general population, ${ }^{7,8}$ approximately 1.5 million women in the United States are also at risk for premutation-associated disorders such as fragile X-associated tremor/ataxia syndrome (OMIM 300623) and fragile $\mathrm{X}$-associated primary ovarian insufficiency (OMIM 300624).

Since the identification of the gene FMR1 in 1991, risk estimates for full mutation expansions have been based on the maternal repeat length. The risk of expansion increases with
AGGs. Two contractions in maternal transmission were accompanied by loss of AGGs, suggesting a mechanism for generating alleles that lack AGG interruptions. Maternal age was examined as a factor in full mutation expansions using prenatal samples to minimize ascertainment bias, and a possible effect was observed though it was not statistically significant $(P=0.06)$.

Conclusion: These results strengthen the association of AGG repeats with CGG repeat stability and provide more accurate risk estimates of full mutation expansions for women with 45-90 repeat alleles.

Genet Med advance online publication 11 September 2014

Key Words: carrier screening; FMR1; fragile X; genetic counseling; trinucleotide repeat instability

increasing repeat length and is influenced by family history of FXS. ${ }^{9}$ More than $94 \%$ of alleles $>90$ CGGs expand to a full mutation, ${ }^{9}$ whereas a 56-repeat allele is the smallest known to expand to a full mutation in one generation. ${ }^{10}$ Today, many pregnant women are screened for their fragile $\mathrm{X}$ carrier status irrespective of family history. In the absence of a family history of fragile $\mathrm{X}$, however, risk assessments are problematic because some newly identified intermediate and small premutation alleles are stably inherited. ${ }^{9}$ In 1994, Eichler et al. ${ }^{11}$ suggested that AGGs interspersed within the FMR1 repeat region increase its stability. In the general population, $94 \%$ of alleles have one or two AGG interruptions, which are most commonly observed on the $5^{\prime}$ end of the repeat tract as the $10^{\text {th }}$ or $11^{\text {th }}$ and $20^{\text {th }}$ or $21^{\text {st }}$ triplet of the repeat. In contrast, alleles in fragile $\mathrm{X}$ families are less likely to include AGGs at the $5^{\prime}$ end, and they contain long stretches of uninterrupted CGGs at the $3^{\prime}$ end. Recent advances in polymerase chain reaction (PCR) technologies have enabled larger cohort studies and examination of AGG status in females. ${ }^{9,12}$ These studies have affirmed the influence of AGG interruptions on instability and risk of full mutation expansion. ${ }^{13,14}$ We previously examined 457 maternal and 81 paternal transmissions for alleles with 45-69 repeats. We observed that alleles with no AGGs exhibited the greatest degree of instability on transmission and that maternal premutation alleles with no AGGs also had the greatest risk of expansion to the full 
mutation. ${ }^{13}$ To better assess the effect of AGG interruptions on repeat instability and full mutation expansion of the maternal allele, we have extended these studies by including nearly twice as many transmissions and by increasing the upper allele size limit from 69 to 90 repeats. These studies provide improved risk estimates for women identified as carrying intermediate and premutation alleles based on repeat length and AGG status.

\section{Subjects}

\section{MATERIALS AND METHODS}

The 705 families in the study were ascertained as follows: 356 from population screening; 178 with a family history of fragile $\mathrm{X}$; 96 from individuals with developmental disabilities of unknown etiology; 4 from individuals with neurological symptoms; 5 with primary ovarian insufficiency; and 64 for whom ascertainment was unknown. The families included 377 previously examined. ${ }^{13}$ Transmissions from male and female carriers with 45-90 repeat alleles in these families were analyzed. In the repeat length category of 45-69, 140 maternal and 12 paternal transmissions had not been included in our previous study. ${ }^{13}$ The results were similar to the previous data set; therefore, we report findings only for the larger data set that includes all samples. Transmissions of maternal alleles $>90$ repeats were not included in this study because nearly all of these expand to full mutations. Purified genomic DNA from the other 328 families was collected under institutional review board approval from the New York State Institute for Basic Research in Developmental Disabilities.

\section{PCR protocol and data analysis}

The AGG interruption pattern within the fragile $\mathrm{X}$ repeat was determined at Asuragen (Austin, TX) for all females and most males using PCR and capillary electrophoresis as previously described. ${ }^{13}$ AGG status in 314 males was defined by Sanger sequencing. The AGG structure within families was consistent regardless of the methodology used. Changes in allele repeat length on transmission were determined by comparison of parental and transmitted alleles in side-by-side PCR capillary electrophoretic analyses. CGG repeat sizing by this methodology was very consistent. Comparison of size calls for identical alleles in maternal DNA samples and fetal samples with maternal cell contamination had an intraclass correlation coefficient of $>0.99 .{ }^{15}$ Size calls of the duplicated alleles in these samples varied by $<1$ bp or $<0.33$ of a CGG repeat (mean difference $=$ $0.313 \pm 0.269 \mathrm{bp}$ ). Thus, inaccuracy of allele size calls did not contribute significantly to the changes in allele size on transmission reported in Results.

An unstable transmission was defined as a change of at least one repeat from parent to child. All analyses were stratified by parental origin of the transmission. Full mutation alleles were further characterized using gene-specific PCR ${ }^{12}$ and FlashGel electrophoresis with $1.2 \%$ precast agarose gels (Lonza, Basel, Switzerland). Full mutation allele sizes were estimated from a Quick-Load 2-Log DNA ladder (New England Biolabs, Ipswich, MA). Mosaic full mutations were defined as the presence of both premutation and full mutation alleles in an individual.

\section{Statistical methods}

We used generalized estimating equation (GEE) methodology $^{16,17}$ to examine the effect of maternal age on risk of instability ( 0 = stable; 1 = unstable) and on the risk of full mutation transmission ( 0 = unstable, not full; $1=$ full mutation). Mothers' repeat sizes and numbers of AGG interruptions were covariates, as was maternal age. Data were grouped by mother and correlation structure set to "exchangeable." All GEE analyses were run on Stata (Release 13, StataCorp, College Station, TX). Analysis of variance models were used to test for mean differences in magnitude of instability by number of AGG interspersions ( 0 , 1 , or $>1$ AGG interspersion). Linear regression using maternal repeat size and number of AGG interruptions as predictors was also used to test for the effects on full mutation repeat size. These analyses were run using SigmaPlot 11.0.

\section{RESULTS}

We have examined the association of AGG interruptions and fragile $\mathrm{X}$ repeat instability for 1,040 transmissions from 726 mothers and 80 fathers in 705 families. Table 1 summarizes full mutation expansions among the 918 transmissions of maternal alleles with 45-90 repeats. The transmissions were grouped into categories of five repeats based on maternal repeat size. A total of 168 (18\%) transmissions expanded to full mutations. The full mutation frequency increased with increasing maternal allele size, as has been observed in other studies. The smallest allele in this cohort to expand to a full mutation had 59 repeats, giving an expansion rate of $0.5 \%(1 / 197)$ for maternal alleles with 55-59 repeats.

\section{Repeat instability and full mutation expansion in maternal alleles}

We next considered the number of AGGs in maternal alleles and their impact on transmission in repeat instability in general and specifically in full mutation expansions. Table 2 shows unstable transmissions and full mutation expansions in the nine maternal repeat size categories subdivided by the number of AGGs. Overall, there were 227 transmissions from maternal alleles with no AGGs, 401 from alleles with one AGG, 272 from alleles with two AGGs, 16 from alleles with three AGGs, and 2 from alleles with four AGGs. Four AGGs were observed only in

Table 1 Full mutation expansions by maternal repeat size

\begin{tabular}{lcl} 
Maternal repeat size & $\begin{array}{c}\text { No. full mutation/ } \\
\text { total transmissions }\end{array}$ & $\%$ \\
\hline $45-49$ & $0 / 98$ & 0 \\
\hline $50-54$ & $0 / 102$ & 0 \\
\hline $55-59$ & $1 / 197$ & 0.5 \\
\hline $60-64$ & $2 / 115$ & 1.7 \\
\hline $65-69$ & $6 / 85$ & 7 \\
\hline $70-74$ & $18 / 84$ & 21 \\
\hline $75-79$ & $47 / 99$ & 47 \\
\hline $80-84$ & $60 / 96$ & 62 \\
\hline $85-90$ & $34 / 42$ & 81 \\
\hline Total & $168 / 918$ & 18 \\
\hline
\end{tabular}


Table 2 Unstable transmissions and full mutation expansions sorted by repeat size and number of AGGs

\begin{tabular}{|c|c|c|c|c|c|c|}
\hline $\begin{array}{l}\text { Maternal repeat } \\
\text { size }\end{array}$ & No. AGGs & Total transmissions & Unstable transmissions ${ }^{a}$ & $\%$ & No. full mutations & $\%$ \\
\hline \multirow{3}{*}{$45-49$} & 1 & 32 & 6 & 19 & 0 & 0 \\
\hline & 3 & 3 & 0 & 0 & 0 & 0 \\
\hline & 4 & 1 & 0 & 0 & 0 & 0 \\
\hline \multirow{3}{*}{$50-54$} & 2 & 41 & 5 & 12 & 0 & 0 \\
\hline & 3 & 2 & 0 & 0 & 0 & 0 \\
\hline & 4 & 1 & 0 & 0 & 0 & 0 \\
\hline \multirow[t]{2}{*}{$55-59$} & 0 & 30 & 29 & 97 & 1 & 3 \\
\hline & 1 & 95 & 50 & 53 & 0 & 0 \\
\hline \multirow{3}{*}{$60-64$} & 1 & 39 & 33 & 85 & 0 & 0 \\
\hline & 2 & 38 & 20 & 53 & 0 & 0 \\
\hline & 3 & 1 & 1 & 100 & 0 & 0 \\
\hline \multirow[t]{4}{*}{ 65-69 } & 0 & 35 & 35 & 100 & 6 & 17 \\
\hline & 1 & 28 & 25 & 89 & 0 & 0 \\
\hline & 2 & 20 & 14 & 70 & 0 & 0 \\
\hline & 3 & 2 & 0 & 0 & 0 & 0 \\
\hline \multirow[t]{3}{*}{ 70-74 } & 0 & 29 & 29 & 100 & 15 & 52 \\
\hline & 1 & 41 & 40 & 98 & 3 & 7 \\
\hline & 2 & 14 & 13 & 93 & 0 & 0 \\
\hline \multirow{2}{*}{$85-90$} & 1 & 30 & 30 & 100 & 25 & 83 \\
\hline & 2 & 4 & 4 & 100 & 2 & 50 \\
\hline
\end{tabular}

${ }^{\mathrm{a} A}$ change of one or more repeats.

intermediate alleles, and three AGGs were found only in alleles with $<70$ repeats.

Maternal alleles with no AGGs had the greatest risk for full mutation expansion. Fifty-three percent (90/168) of the full mutation expansions occurred from maternal alleles with no AGG interruptions, and 43\% (72/90) occurred from maternal alleles with one AGG. Only 4\% (6/190) of full mutation expansions occurred from maternal alleles with two AGGs. The relationship of AGG structure to full mutation expansion was consistent in the different repeat size classes: $89 \%$ $(24 / 27)$ of maternal premutation alleles with $<75$ repeats that expanded to full mutations included no AGGs, and the remaining $11 \%$ (3) had only one. Alleles with $>74$ repeats showed a similar pattern-those with no AGGs had the highest rate of expansion to the full mutation, followed by alleles with one, and then by those with two. Supplementary Figure S1 online highlights the influence of AGGs on the percentage of full mutation expansions observed within this repeat range of 45-90.

\section{Repeat instability in paternal and maternal alleles}

To understand the risk and magnitude of repeat instability for alleles that did not expand to a full mutation, we compared transmissions from both maternal and paternal alleles. Table 3 summarizes outcomes of unstable maternal and paternal transmissions. Sixty-six percent of maternal and paternal alleles changed by one or more repeats in transmission. For transmissions from alleles with $<70$ repeats, paternal alleles had a greater likelihood for instability than did maternal alleles (Fisher exact test $P=0.046)$. For larger alleles $(70-90$ repeats), maternal 
Table 3 Unstable maternal and paternal transmissions

\begin{tabular}{|c|c|c|c|c|}
\hline \multirow{2}{*}{$\begin{array}{l}\text { Parental } \\
\text { repeat } \\
\text { size }\end{array}$} & \multicolumn{2}{|l|}{ Maternal } & \multicolumn{2}{|l|}{ Paternal } \\
\hline & $\begin{array}{l}\text { No. unstable/total } \\
\text { transmissions }\end{array}$ & $\%$ & $\begin{array}{l}\text { No. unstable/total } \\
\text { transmissions }\end{array}$ & $\%$ \\
\hline $45-49$ & $13 / 98$ & 13 & $4 / 20$ & 20 \\
\hline $50-54$ & $25 / 102$ & 24 & $6 / 13$ & 46 \\
\hline $55-59$ & $85 / 197$ & 43 & $14 / 25$ & 56 \\
\hline $60-64$ & $89 / 115$ & 78 & $17 / 20$ & 85 \\
\hline $65-69$ & $74 / 85$ & 87 & $14 / 15$ & 93 \\
\hline 70-74 & $82 / 84$ & 98 & $2 / 3$ & 67 \\
\hline 75-79 & 99/99 & 100 & $6 / 7$ & 86 \\
\hline $80-84$ & $96 / 96$ & 100 & $12 / 13$ & 92 \\
\hline $85-90$ & $42 / 42$ & 100 & $6 / 6$ & 100 \\
\hline Total & $606 / 918$ & 66 & $81 / 122$ & 66 \\
\hline
\end{tabular}

transmissions were more often unstable (Fisher exact test $P=$ 0.004).

The range of repeat size changes for unstable paternal and maternal transmissions excluding full mutation expansions is shown in Table 4. For both maternal and paternal transmissions, the largest changes observed were for alleles with no AGGs. Only 35\% (93/266) of maternal alleles with two AGGs changed on transmission compared with 98\% (134/137) for alleles lacking an AGG. Fourteen percent (14/102) of maternal transmissions with 50-54 repeats expanded to premutation size alleles. Seven of these contained no AGGs, four had one AGG, and three had two AGGs. Although most of the expansions had increases of only one to four repeats, larger increases were observed for maternal alleles with no AGGs, including one that expanded from 54 to 85 repeats in the next generation. For alleles with 55-69 repeats, the median repeat size change for alleles with no AGGs compared with those with two AGGs differed by as much as 10-fold. The majority of parental alleles with $>70$ repeats were unstable and, excluding expansion to a full mutation, had similar changes regardless of AGG status.

\section{Assessment of CGG repeat contractions}

Twenty-eight contractions were observed among the 1,040 transmissions: $2.3 \%(21 / 918)$ of maternal transmissions and $5.7 \%$ (7/122) of paternal transmissions (Table 5). For parental alleles with 45-69 repeats, contractions occurred more frequently in paternal transmissions than in maternal transmissions, although this trend did not reach the 0.05 level of significance. For larger alleles with 70-90 repeats, paternal transmissions were four times as likely to undergo contractions as maternal transmissions (Fisher exact test $P=0.027$ ). The AGG structures of parents and offspring are shown in Supplementary Table S1 online. There was no apparent association between specific AGG structures and contractions. In total, eight of the parental alleles had no AGGs, 12 had one AGG, and six had two AGGs. With one exception, all of the alleles had relatively long stretches ( $>36$ ) of uninterrupted CGG repeats at the $3^{\prime}$ end. The paternally transmitted contractions had an average loss of five repeats, whereas the maternally transmitted contractions had an average loss of 10 repeats. There were also two unrelated mothers who each had two children who inherited contractions. Two contractions of maternal alleles were accompanied by a loss of AGGs. In one, a maternal allele with 65 repeats and two AGGs contracted to 45 repeats, losing both AGGs. In a second, a maternal allele with 78 repeats and one AGG contracted to 61 repeats with no AGG. No loss of AGGs occurred in contractions of paternal alleles.

\section{Assessment of maternal age on repeat expansion}

We examined the effect of maternal age on the risk of expansion to the full mutation using GEE with maternal allele size, number of maternal AGG interruptions, and maternal age as predictors. To minimize ascertainment bias, we performed an analysis of the prenatal samples only. The analysis of 291 prenatal transmissions from 257 mothers with 55-90 repeats showed that both maternal allele size and the number of AGGs were highly significant predictors $(P<0.001)$; however, maternal age showed only a possible association (odds ratio $=1.11$, 95\% confidence interval: $0.99-1.24 ; P=0.06)$ with full mutation transmission. We also examined sibships from this data set with more than one transmission of the at-risk allele: in 15 there were only premutations, in 11 there were only full mutations, and in 2 there were both premutation and full mutation offspring. In the last group with both premutation and full mutation offspring, one mother transmitted a full mutation allele at a younger age, whereas the second mother transmitted a full mutation at an older age. In a separate analysis, we looked at the effect of maternal age on stable compared with unstable transmissions in mothers with $45-65$ repeats. This size category was selected to minimize the influence of full mutation transmissions. Analysis of 419 transmissions from 363 mothers showed both maternal allele size and number of AGG interruptions were highly significant predictors $(P<0.001)$, but only a possible effect was seen with maternal age (odds ratio $=1.05,95 \%$ confidence interval: $0.999-1.10 ; P=0.056)$.

\section{Size and mosaicism in full mutation alleles}

We also considered the impact of AGG interruptions on the magnitude of full mutation expansions and the occurrence of mosaic full mutations. Size estimates of full mutation alleles were obtained for 134 of 168 full mutations. Most full mutations were characterized by multiple peaks in the full mutation range, observed either by capillary or by agarose gel electrophoresis. The median expansion was $400 \mathrm{CGGs,}$ with a range from 210 to 1,300 CGG. The magnitude of expansion was not normally distributed. No significant differences in magnitude were observed with no or one AGG, nor was there a significant association with maternal repeat length. Mosaic full mutations with both premutation and full mutation alleles in the same individual were observed in $38 \%(51 / 134)$ of full mutations. Of these, 59\% were from maternal alleles with one AGG, compared with $37 \%$ of samples with no AGG. The difference in mosaicism attributable to the number of AGGs was significant $(P<0.03)$. 
Table 4 Range of repeat change in unstable paternal and maternal transmissions excluding full mutation expansions

\begin{tabular}{|c|c|c|c|c|c|c|c|}
\hline \multirow[b]{2}{*}{$\begin{array}{l}\text { Parental } \\
\text { repeat } \\
\text { size }\end{array}$} & \multirow[b]{2}{*}{$\begin{array}{l}\text { No. } \\
\text { AGGs }\end{array}$} & \multicolumn{3}{|c|}{ Maternal } & \multicolumn{3}{|c|}{ Paternal } \\
\hline & & $\begin{array}{l}\text { No. unstable/ } \\
\text { total } \\
\text { transmissions }\end{array}$ & Range of repeat change & $\begin{array}{l}\text { Median } \\
\text { repeat } \\
\text { change }\end{array}$ & $\begin{array}{l}\text { No. unstable/ } \\
\text { total } \\
\text { transmissions }\end{array}$ & Range of repeat change & $\begin{array}{l}\text { Median } \\
\text { repeat } \\
\text { change }\end{array}$ \\
\hline \multirow{3}{*}{$45-49$} & 1 & $6 / 32$ & $1-2$ & 1 & $2 / 10$ & $1-2$ & 1.5 \\
\hline & 2 & $3 / 57$ & -1 to 2 & 1 & $1 / 8$ & 1 & 1 \\
\hline & 3 & $0 / 3$ & 0 & - & $0 / 1$ & 0 & - \\
\hline \multirow[t]{5}{*}{$50-54$} & 0 & $9 / 9$ & $1-31$ & 4 & $2 / 2$ & $2-3$ & 2.5 \\
\hline & 1 & $11 / 49$ & -20 to 4 & 1 & $3 / 4$ & $1-2$ & 1 \\
\hline & 2 & $5 / 41$ & $1-4$ & 2 & $1 / 6$ & 1 & 1 \\
\hline & 3 & $0 / 2$ & 0 & - & $0 / 1$ & 0 & - \\
\hline & 4 & $0 / 1$ & 0 & - & - & - & - \\
\hline $55-59$ & 3 & $0 / 8$ & 0 & - & $0 / 1$ & 0 & - \\
\hline \multirow[t]{4}{*}{$60-64$} & 0 & $34 / 35$ & 4-93 & 16 & $15 / 16$ & $2-54$ & 11 \\
\hline & 1 & $33 / 39$ & -4 to 10 & 4 & $1 / 1$ & 7 & 7 \\
\hline & 2 & $20 / 38$ & -17 to 24 & 1 & $1 / 3$ & -2 & -2 \\
\hline & 3 & $1 / 1$ & 2 & 2 & - & - & - \\
\hline \multirow[t]{4}{*}{$65-69$} & 0 & $29 / 29$ & -9 to 68 & 20 & $11 / 11$ & -2 to 11 & 4 \\
\hline & 1 & $25 / 28$ & $2-48$ & 8 & $3 / 4$ & -1 to 11 & 3 \\
\hline & 2 & $14 / 20$ & $1-4$ & 1.5 & - & - & - \\
\hline & 3 & $0 / 2$ & 0 & - & - & - & - \\
\hline $70-74$ & 0 & $14 / 14$ & -11 to 70 & 35.5 & $2 / 3$ & $10-13$ & 11.5 \\
\hline $80-84$ & 2 & $17 / 17$ & $4-83$ & 15.5 & $1 / 1$ & 5 & 5 \\
\hline \multirow[t]{3}{*}{$85-90$} & 0 & $1 / 1$ & 59 & 59 & $1 / 1$ & -8 & -8 \\
\hline & 1 & $5 / 5$ & $30-87$ & 74 & $4 / 4$ & -4 to 35 & 6 \\
\hline & 2 & $2 / 2$ & $10-13$ & 11.5 & $1 / 1$ & 40 & 40 \\
\hline
\end{tabular}

Table 5 Summary of contractions in maternal and paternal transmissions

\begin{tabular}{|c|c|c|c|c|}
\hline \multirow{2}{*}{$\begin{array}{l}\text { Parental } \\
\text { repeat } \\
\text { size }\end{array}$} & \multicolumn{2}{|l|}{ Maternal } & \multicolumn{2}{|l|}{ Paternal } \\
\hline & $\begin{array}{l}\text { No. contractions/ } \\
\text { transmissions }\end{array}$ & $\%$ & $\begin{array}{l}\text { No. contractions/ } \\
\text { transmissions }\end{array}$ & $\%$ \\
\hline $45-69$ & $10 / 597$ & 1.7 & $3 / 93$ & 3.2 \\
\hline 70-90 & $11 / 321$ & 3.4 & $4 / 29$ & 13.8 \\
\hline Total & $21 / 918$ & 2.3 & $7 / 122$ & 5.7 \\
\hline
\end{tabular}

\section{DISCUSSION}

The increased awareness of FXS and relative frequency of FMR1 premutation alleles and the development of PCR-based technologies have driven an increase in population-based carrier testing. This has led to the identification of women as carriers without a diagnosis of FXS in their families. Because many of the newly identified maternal alleles are stable on transmission, assigning full mutation expansion risk has been difficult. Since detection of the FMR1 gene in 1991, expansion risks have been based on maternal repeat length alone. However, evidence for the role of interspersed AGGs in stability of the CGG repeat region has accumulated over the past 20 years. Recently, larger cohort studies have affirmed the association of AGG with risk of expansion from maternal alleles. Yrigollen et al. ${ }^{14}$ showed that AGG elements substantially modified the risk of a full mutation expansion from a given repeat length. However, the limited size of the cohort and the potential bias in its ascertainment made it prudent to follow up these results with further studies. In previous work, we defined the role of AGG in instability in the range of 45-69 repeats. ${ }^{13}$ We found a 19-fold difference for alleles with no AGGs compared with those with two, and we observed 
that all full mutation expansions were transmitted from alleles lacking AGGs. However, that study was limited in the repeat range evaluated. Here, we extended our previous study and investigated the AGG structure in an additional 328 families. Furthermore, the repeat sizes analyzed ranged from 45 to 90 repeats as compared with the previous study of 45-69 repeats. This large cohort of 1,040 transmissions allowed a comparison of instability for maternal and paternal transmissions and strengthened the risk estimates for full mutation expansions with particular regard to AGG structure. Our current study also allowed a careful examination of contractions observed in both maternal and paternal transmissions and suggested that contractions may be a common path for loss of AGGs.

The presence of even a single AGG significantly reduced the risk of full mutation expansions for alleles with $<80$ repeats. This effect is most dramatic for alleles $<70$ repeats. As the total repeat length increases beyond 70 , the allele instability is substantial despite the presence of the AGG interruptions they may contain. Once the repeat length exceeds 90 repeats, there is no apparent effect of AGG interruptions, and it is clear from other studies that nearly all of these maternal alleles expand to the full mutation. We also considered the risk of instability and magnitude of change for the 750 alleles that did not expand to a full mutation (Table 4). For alleles with $<70$ repeats, the presence of two AGGs appears to limit the instability on transmission. Thus, even in the absence of full mutation expansions, AGG analysis should be offered as an option because it provides valuable information and helps identify alleles at higher risk for instability in future generations.

Using GEE methodology, we have shown that total repeat length and number of AGGs are significant predictors of instability. Other studies have generated a similar model using separate cohorts and different statistical methodology. ${ }^{14}$ Because of the suggestion by Sherman et al. ${ }^{18}$ that maternal age may be a risk factor in instability, we examined this question. Although GEE suggested a positive association between maternal age and risk of instability, this association did not reach significance at the 0.05 level. Because maternal allele size and AGG structure are the most important predictors, the effects of maternal age, even if substantiated by additional studies, would have only a small impact on risk estimates.

Unlike expansions, contractions do not appear to be affected by AGG structure within the repeat. That is, contractions for both maternal and paternal transmissions include alleles with none, one, or two AGGs, suggesting that contractions result from a different mechanism than expansions. Genetic factors may play a role, because we did observe two unrelated women, each of whom had two children with contractions in size. For fathers, larger repeat lengths are associated with an increased likelihood to contract on transmission. We observed that $3.2 \%$ of paternal transmissions with $45-$ 69 repeats and $13.8 \%$ of transmissions with $70-90$ repeats underwent contractions (Table 5). In additional studies not reported here (unpublished data), 36\% (12/33) of paternal alleles with $>90$ repeats contracted on transmission. Although there were more contractions among maternal alleles in our study, a greater percentage of contractions occurred in paternal transmissions. Previous studies have documented contractions in males with normal ${ }^{19,20}$ and premutation alleles. ${ }^{21,22}$ Furthermore, although males with full mutations have full mutation alleles in their somatic cells, only premutation size alleles are present in sperm. ${ }^{23}$ This is in stark contrast to full mutation expansions that occur, with increasing likelihood in female transmissions as allele size increases.

The only loss of AGGs in 1,040 transmissions occurred in two maternal alleles that contracted in size. No loss of AGGs was seen in the other transmissions that included 659 expansions and 353 stable alleles. In additional studies not reported here (unpublished data), we have also observed a 94 repeat maternal allele that lost one of two AGGs on contraction to 34 repeats. Although loss of AGGs was not seen in contractions of paternal alleles, this was probably attributable to the limited number of paternal transmissions. These findings suggest that the primary mechanism for AGG loss is through contraction to a smaller size. Point mutations of $\mathrm{C}$ to $\mathrm{A}$ were not observed in our study and have not been reported elsewhere.

It is interesting to note that few expanded alleles had more than two AGGs. Two intermediate alleles contained four AGGs, and 16 alleles with $<70$ repeats contained three AGGs. In general these were highly stable, although one maternal allele with 61 repeats and three AGGs increased to 63 in her offspring. How do alleles with multiple AGGs arise? There is one report in the literature of a paternal 29-repeat allele with two AGGs expanding in one generation to a 39-repeat allele with three AGGs. ${ }^{24}$ The addition of a cassette of nine CGGs and one AGG may be a possible path to these alleles.

This work should allow counselors to give more accurate risk estimates to women with 45-90 repeat alleles. Alleles with no AGG interruptions had the greatest risk for unstable transmissions. Although maternal alleles with $45-49$ repeats and no AGGs may be unstably inherited, the increases are small and there is no apparent risk of full mutation expansion in a single transmission. Thus, prenatal testing or analysis of other family members is considered unnecessary at this time. For maternal alleles with 50-54 repeats, there is a risk for expansion into the premutation range, but no apparent risk for full mutation expansion in the next generation. Although prenatal analysis is not necessarily indicated, some women with these alleles may nevertheless be interested in prenatal and family testing to identify the inheritance of premutation alleles. Because women who carry alleles with $>55$ repeats and no AGGs have a risk of full mutation expansion in one generation, they should be offered the option of fragile X prenatal studies. Maternal alleles with one or two AGGs carry risks for instability and may also be at risk for full mutation expansions depending on their size. Here, decisions about prenatal testing should be made on an individual basis.

To summarize, this study and others ${ }^{13,14}$ firmly establish that analysis of the AGG structure within the FMR1 repeat identifies alleles at greatest risk for full mutation expansion. Thus, 
women who know that their premutation alleles lack AGGs can make informed decisions about whether to undergo fragile $\mathrm{X}$ prenatal testing. In contrast, women who know that they are at reduced risk for full mutation transmissions because their premutation alleles have two or more AGGs may choose to avoid the risk and expense associated with invasive testing for their pregnancies. Our studies also suggest repeat contraction and loss of AGGs as a pathway to greater instability, expansion, and the risk of FXS. Nevertheless, although FMR1 repeat length and the absence of AGG interruptions are major contributors to repeat expansion, other factors such as allelic variation in components of DNA replication and repair systems are likely to have a role as well.

\section{SUPPLEMENTARY MATERIAL}

Supplementary material is linked to the online version of the article at $h$ ttp://www.nature.com/gim

\section{ACKNOWLEDGMENTS}

We thank the families whose support made this work possible. We also thank Jeffrey Taylor and Jennifer McCarver at Asuragen for preparing samples for analysis. Special thanks go to Michael Flory and Stephanie L. Sherman for assistance in statistical analyses. This work was supported in part by the New York State Institute for Basic Research in Developmental Disabilities and the New York State Office of People With Developmental Disabilities, and by an award from the Eunice Kennedy Shriver National Institute of Child Health and Human Development (5R44HD066953-03 to A.G.H. and G.J.L.).

\section{DISCLOSURE}

A.G.H., G.J.L., and S.S. are full-time employees of and have stock options in Asuragen, Inc. The other authors declare no conflict of interest.

\section{REFERENCES}

1. Pearson CE, Nichol Edamura K, Cleary JD. Repeat instability: mechanisms of dynamic mutations. Nat Rev Genet 2005;6:729-742.

2. Oberlé I, Rousseau F, Heitz D, et al. Instability of a 550-base pair DNA segment and abnormal methylation in fragile X syndrome. Science 1991;252:10971102.

3. Verkerk AJ, Pieretti M, Sutcliffe JS, et al. Identification of a gene (FMR-1) containing a CGG repeat coincident with a breakpoint cluster region exhibiting length variation in fragile X syndrome. Cell 1991;65:905-914.

4. Yu S, Pritchard M, Kremer E, et al. Fragile X genotype characterized by an unstable region of DNA. Science 1991;252:1179-1181.

5. Colak D, Zaninovic N, Cohen MS, et al. Promoter-bound trinucleotide repeat $m R N A$ drives epigenetic silencing in fragile $X$ syndrome. Science 2014;343:1002-1005.

6. Nolin SL, Brown WT, Glicksman A, et al. Expansion of the fragile X CGG repeat in females with premutation or intermediate alleles. Am J Hum Genet 2003;72:454-464.
7. Cronister A, Teicher J, Rohlfs EM, Donnenfeld A, Hallam S. Prevalence and instability of fragile $X$ alleles: implications for offering fragile $X$ prenatal diagnosis. Obstet Gynecol 2008;111:596-601.

8. Seltzer MM, Baker MW, Hong J, Maenner M, Greenberg J, Mandel D. Prevalence of carriers of premutation-size alleles of the FMR1 gene and implications for the population genetics of the fragile X syndrome. Am J Med Genet B Neuropsychiatr 2012; 159:589-597.

9. Nolin SL, Glicksman A, Ding X, et al. Fragile X analysis of 1112 prenatal samples from 1991 to 2010. Prenat Diagn 2011;31:925-931.

10. Fernandez-Carvajal I, Lopez Posadas B, Pan R, Raske C, Hagerman PJ, Tassone F. Expansion of an FMR1 grey-zone allele to a full mutation in two generations. $J$ Mol Diagn 2009;11:306-310.

11. Eichler EE, Holden JJ, Popovich BW, et al. Length of uninterrupted CGG repeats determines instability in the FMR1 gene. Nat Genet 1994;8:88-94.

12. Chen L, Hadd A, Sah S, et al. An information-rich CGG repeat primed PCR that detects the full range of fragile $X$ expanded alleles and minimizes the need for southern blot analysis. J Mol Diagn 2010;12:589-600.

13. Nolin $S L$, Sah $S$, Glicksman A, et al. Fragile X AGG analysis provides new risk predictions for 45-69 repeat alleles. Am J Med Genet A 2013;161A:771-778.

14. Yrigollen $C M$, Durbin-Johnson $B$, Gane L, et al. AGG interruptions within the maternal FMR1 gene reduce the risk of offspring with fragile $\mathrm{X}$ syndrome. Genet Med 2012;14:729-736.

15. Shrout PE, Fleiss JL. Intraclass correlations: uses in assessing rater reliability. Psychol Bull 1979;86:420-428.

16. Liang KY, Zeger SL. Longitudinal data analysis using generalized linear models. Biometrika 1986;73:13-22.

17. Zeger SL, Liang KY. Longitudinal data analysis for discrete and continuous outcomes. Biometrics 1986;42:121-130.

18. Sherman SL, Meadows KL, Ashley AE. Examination of factors that influence the expansion of the fragile $\mathrm{X}$ mutation in a sample of conceptuses from known carrier females. Am J Med Genet 1996;64:256-260.

19. Crawford DC, Wilson B, Sherman SL. Factors involved in the initial mutation of the fragile X CGG repeat as determined by sperm small pool PCR. Hum Mol Genet 2000;9:2909-2918.

20. Sullivan AK, Crawford DC, Scott EH, Leslie ML, Sherman SL. Paternally transmitted FMR1 alleles are less stable than maternally transmitted alleles in the common and intermediate size range. Am J Hum Genet 2002;70:15321544.

21. Nolin SL, Lewis FA 3rd, Ye LL, et al. Familial transmission of the FMR1 CGG repeat. Am J Hum Genet 1996;59:1252-1261.

22. Nolin SL, Houck GE Jr, Gargano AD, Blumstein H, Dobkin CS, Brown WT. FMR1 CGG-repeat instability in single sperm and lymphocytes of fragile-X premutation males. Am J Hum Genet 1999;65:680-688.

23. Reyniers E, Vits L, De Boulle K, et al. The full mutation in the FMR-1 gene of male fragile X patients is absent in their sperm. Nat Genet 1993;4:143-146.

24. Macpherson JN, Curtis G, Crolla JA, et al. Unusual (CGG) expansion and recombination in a family with fragile X and DiGeorge syndrome. J Med Genet 1995;32:236-239.

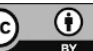

This work is licensed under a Creative Commons Attribution 3.0 Unported License. The images or other third party material in this article are included in the article's Creative Commons license, unless indicated otherwise in the credit line; if the material is not included under the Creative Commons license, users will need to obtain permission from the license holder to reproduce the material. To view a copy of this license, visit http:// creativecommons.org/licenses/by/3.0/ 